\title{
A study on the vibration-based self-monitoring capabilities of nano-enriched composite laminated beams.
}

\author{
David García $^{1}$, Irina Trendafilova ${ }^{1}$ and Daniel J Inman ${ }^{2}$ \\ ${ }^{1}$ Mechanical \& Aerospace Engineering, University of Strathclyde, 75 Montrose street, G11XJ, \\ Glasgow, UK \\ ${ }^{2}$ Aerospace Engineering, University of Michigan, 1320 Beal Ave, 48109-2140, Ann Arbor, MI, \\ USA \\ E-mail: david.garcia@strath.ac.uk
}

\begin{abstract}
.
This is an exploratory investigation on the self-sensing capabilities of nano-enriched glass/fibre laminates for damage detection purposes through changes in the dynamic responses, which are estimated by measuring the changes in voltage due to a dynamic strain. The deformation of the nano-enriched structure introduces changes in the resistance/voltage of the nanocomposites. The measured voltage signals contain information of the vibratory response of the laminated beam. This research uses a vibration-based data driven methodology for damage detection applied for the estimated vibratory signals using the conductivity properties of the embedded nano-particles. The structure considered in this study is a glass/fibre laminated beam enriched with carbon black nanoparticles (CB). The structure is subjected to a direct electric current and the voltage signal is measured. The vibration based monitoring method used is generally based on singular spectrum analysis applied on the estimated vibratory response. The voltage response signal is divided into a certain number of principal components which contain the oscillatory components distributed by their content of variance in the voltage signal. The components with more variance are used to define a reference state based on the status of the healthy structure. Consequently, the estimated vibratory signals from beams with a simulated damage are compared to the healthy state which eventually results in the damage detection procedure. The damage was simulated firstly by adding an additional mass on the beam tip and secondly by drilling a hole on the beam tip. The results demonstrate the potential for using the voltage estimated vibratory signals for self-sensing damage detection purposes in carbon nano-enriched glass/fibre structures.
\end{abstract}

\section{Introduction}

Composite laminated structures are continuously gaining more importance and their applications are constantly growing as a result of their advantageous properties, most notably their highest strength-to-weight ratio, corrosion resistance, high impact strength and their magnificent design flexibility. They are steadily replacing traditional structures in a wide range of industry sectors, including the aerospace, wind energy, marine, and oil and gas industries and even in every day structures. Structural health monitoring (SHM) plays an important role in this kind of structures due to the complexity for modelling the possible failure mechanisms. Micro-crack formations occur in the material matrix and can growth to delamination which in turn can 
reduce the stiffness of the material section up to $60 \%$ without any significant visual change and eventually this can lead to the collapse of the entire structural member. Contemporary research is focused on embodying intelligence in structures and in the development of integrated systems capable to monitor the health and integrity of the structure. The study of smart structures with self-sensing capabilities and real time monitoring capabilities oriented towards the structural integrity is in constant development. The idea of embedding conductive nano-inclusions within the matrix of composite materials to control their conductivity properties and as result their dynamic/vibratory characteristics, contains a lot of potential for the purposes of structural health monitoring. Previous research has focuses on monitoring and an analysis of the electrical resistance to detect damage existence $[1,2]$.

Nanocomposites are experiencing one of the most exciting developments in the current research. They are at the forefront of the contemporary research because of their wide variety of additional functionalities which show promise for applications at many diverse sectors. The use of nanocomposites have aroused great interest to the researches because of their remarkable properties at nanoscale which improve dramatically micro and macro properties such as electromechanical [3], piezorestivity [4, 5] and other mechanical properties [6]. Carbon nanocomposites possess remarkable electrical conductivity properties which can be related to the mechanical properties. Some studies have demonstrated that changes on the strain of the material are transformed in an increment of the resistance [7]. In such a way, monitoring the electrical resistance can be correlated to changes in the mechanical properties of the structure[8]. Damage introduces changes in the electrical conductivity and hence in the resistance. Monitoring the electrical resistance and its changes can be used for the purposes of damage detection and localisation within the boundaries of the specimen analysed [9]. Tallman et al. use an imaging technique known as electrical impedance tomography (EIT) to provide real time monitoring on glass/fibre enriched with CB nanoparticles. This technique has also been applied for thin films made of carbon nanotubes (CNT) [10] or in GFRP manufactued with nanocomposite coatings [11]. Most of the studies uses two or four point probe measurements [12] to obtain the static loads changes. Moreover, many studies are focused on the sensor behaviours under tensile strains and compressive strains [13]. Pham et al. [14] evaluate the sensitivity of the nanocomposite film sensors to show the relationship between CNT and volume fraction and sensitivity. Loh et al. [12] study a single-walled carbon nanotube (SWNT)-polyelectrolyte composite thin film strain sensor fabricated with a layer-by-layer process. And Kang et al. [8] compares the dynamic strain response measured by a laser vibrometer and by a CNT strain sensor. It is clear that carbon nano-inclusions can be used as conductivity/resistance sensors. The material conductivity is related to a number of other mechanical properties, which are in turn related to presence of damage. The question is to use these relations to develop self-health monitoring methods.

Vibration-based structural health monitoring (VSHM) has gained a lot of attention during the last several decades and had demonstrated a vast potential for real time structural health monitoring for a big variety of structures made of different materials [15]. Recently a lot of research has focused on the development of VSHM methods for structures made of composite materials $[16,17]$ but there is still a gap regarding the application of VSHM for self-sensing purposes within nano-carbon enriched materials. This study focuses on a particular application of CB nano-particles enriched composite glass laminated beams and the use of a data-driven VSHM monitoring procedure applied on the measured current signal for the purposes of damage detection.

Nanocomposites sensors are very sensitive to external conditions such as environment temperature and pressure changes or other external factors which cannot be controlled. For this reason an appropriate VSHM methodology is required which can take into account the effects of environmental changes. Modelling the dynamic behaviour of structures with nano-inclusions is 
rather complex task and taking into account the presence of failure/damage make it still more complicated. The data driven methodologies for damage assessment offer a more convenient approach for such kind of problems since they do not assume a model. These non-model based damage assessment methods make use of the measured signal in order to extract information for the structural dynamics and hence its damage state. The most straightforward data-driven methods utilises the measured natural frequencies of the structure and assume that deviations of these frequencies are caused by damage [18]. However, it has been demonstrated that changes on the natural frequencies not always can be associate with damage [19] or in other words changes in the natural frequencies not always are a good indicator for damage. For this reason methods which are purely based on data analysis are gaining popularity $[20,21,22]$ as they use time domain structural vibratory responses which contains all rotational patterns of the dynamic behaviour. Principal component analysis (PCA) methods offer one of such possibilities. The method applied in this study is based on Singular Spectrum Analysis (SSA) which is an extension of PCA for non-independent data $[23,24]$ such as time series. It decomposes the original signal in a certain number of components depending on the unfolded dimension of its window size. These components, the Principal Components (PCs), contain information about the original signal in terms of its variance and the first several ones are responsible for the most of the variability of the vibration response. Eventually, the new PCs are used to obtain the Reconstructed Components ( $\mathrm{RCs}$ ) which can be considered as estimated representation of the healthy structure (reference) and then compare with the damage structure (observation).

The contribution and the novelty of this study is mainly in the extraction of estimated vibratory responses from a nano-enriched glass/fibre beam by measuring changes in voltage due to changes in the mechanical properties of the structure. As explained above a great number of investigations have studied the electrical changes in nano-enriched structures for damage detection but these are mainly based on the loss of conductivity when the damage occurs. Jandro et al. [7] does delamination identification using a quasi-static loading test with a nanocomposite sensor thread. Delamination is identified by the sudden decrease of the load in the loaddeflection curve, and by the jump to infinity of the resistance in the resistancedeflection curve, which corresponds to breaking of one of the sensor threads. However a few studies have explored the analysis of the vibration response of the structure from measurement of the electrical properties. Kang et al. [8] analysed the piezoresistive effect for carbon nanotube polymer strain sensor measuring the vibratory response in a cantilever beam. The study compares the vibratory responses form a healthy and damage beam. The aim of the present investigation aligns in the study of the vibratory response of nano-enriched structures by considering its piezoresistive properties. In our study we do not use a sensor, but the material itself acts as a sensor and the vibration response is measured through the nano-particles which are distributed along the entire specimen. Consequently, the vibratory responses are processed by a vibration-based structural health monitoring methodology for the extraction of useful information which eventually results in the self-damage assessment capability of the laminated beam.

The paper is organised as follows. First the material and laminated beam manufacturing process is explained in $\S 2$. Then, the study concentrates on the electrical and piezoresistivity properties of the nanocomposites. $\S 4$ introduces the vibration-based damage assessment used for the analysis of the voltage signal responses. The description of the experimental setup for a glass/fibre beam with CB nano-particles layers follows. Two damage scenarios are considered: 1) damage is simulated by adding an additional masses on the tip of the beam (5\% and $10 \%$ of the total mass respectively) and 2) damage is introduced by drilling a hole on the tip of the beam $(2 \mathrm{~mm}$ and $4 \mathrm{~mm}$ diameter respectively). $\S 6$ introduces the results and offers a discussion on their interpretation. The paper ends with some conclusion which can be drawn from this 
exploratory study.

\section{Material and laminates manufacturing process}

A glass fibre/epoxy laminated beam is manufactured by hand lay-up and reinforced using stitched unidirectional E-glass with $225 \mathrm{~g} / \mathrm{m}^{2}$ area weight [9]. Epon 8132 epoxy resin with bisphenol-A-based epoxide diluted with alkyl glycidyl ether and JEFFAMINTE T-403 polyetheramine curing agent compounds the matrix material. The mix ratio of the epoxide to curing agent is 100 : 40 by weight. Due to their high structure clusters concentration, Cabot Black Pearls $2000 \mathrm{CB}$ are chosen as nanofillers. The CB nano-particles with high structure clusters have an elongated shape which facilitates the formation of percolate electrical networks at low filler volume fractions [25]. The CB nano-particles are uniformly dispersed in epoxide by a magnetic stirrer and a sonication bath. The CB mixture is firstly magnetically stirred in epoxide for 15 minutes at $250 \mathrm{rpm}$ and secondly is mixed for 4 hours in an ultrasonic bath operating at $45 \mathrm{kHz}$ and $55 \mathrm{~W}$ average power. Finally the mixture is stirred for an additional 15 minutes at $250 \mathrm{rpm}$. BYK A-501 air release and curing agent are subsequently added to the mixture and stirred by hand during 5 minutes. Eventually the mixture is degassed for another 30 minutes. The concentration of $\mathrm{CB}$ in epoxide/curing agent mixture is $0.5 w t \%$. A total of 26 layers are used to produce $4 \mathrm{~mm}$ thickness of the beam. Each layer was impregnated with the CBepoxide using a hand roller (see Figure 1). The staking sequence of the laminates is $\left[[0 / 90]_{6} / 0\right]_{s}$. For the curing process the lay-up laminates are placed over aluminium foil electrodes (top and bottom) in order to apply an alternating current (AC) field to the laminate while the matrix is uncured. This field polarizes and links the highly conductive of CB nano-particles through the thickness direction via dielectrophoresis [26]. As a results of the polarization the laminates are nearly electrically isotropic in the three directions. Although within an individual laminate layer, the conductivity can be several orders of magnitude higher along the fiber direction than perpendicular to the fibers, the equally numbers of layers at $0^{\circ}$ and $90^{\circ}$ approximate an isotropic in-plane conductivity. The field parameters are defined as $1000 \mathrm{~V} / \mathrm{cm}$ and $1 \mathrm{kHz}$ based on [26]. The AC electric field is applied meanwhile the curing process. The parameters of the curing recipe are selected as follows: 30 minutes at $65^{\circ} \mathrm{C}, 2$ hours at $80^{\circ} \mathrm{C}$ and 3 hours at $125^{\circ} \mathrm{C}$. Once the laminates are cured, the edges are cut and sanded in order to guarantee the exposure of the CB mixture. The final dimensions of the beams are $120 \times 12 \times 4 \mathrm{~mm}$.

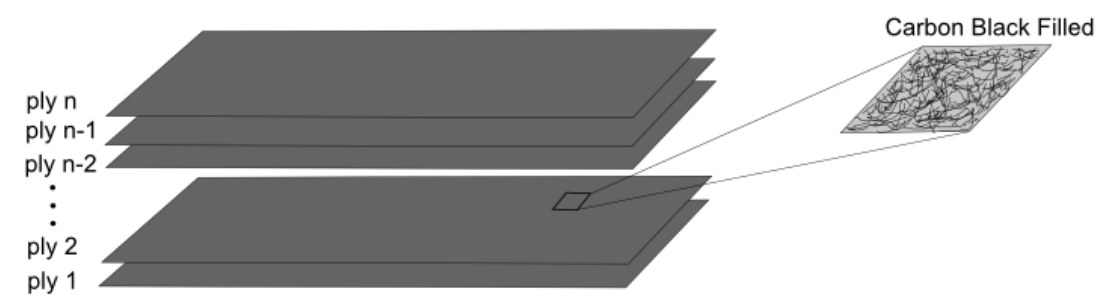

Figure 1. Cabot Black Pearls 2000 CB particles are impregnated in each glass laminated layer by a hand roller.

\section{Electrical conductivity and piezoresistivity of the carbon nano-enriched beam}

High structure CB nano-particles embedded between the laminates generate elongated clusters which facilitate the electrical conductivity [27]. Once the CB-nanoparticles are connected the electrical conductivity occurs due to the mechanical contact between the conductive particles. However, when there is no mechanical contact the electrical connection is realised by the 


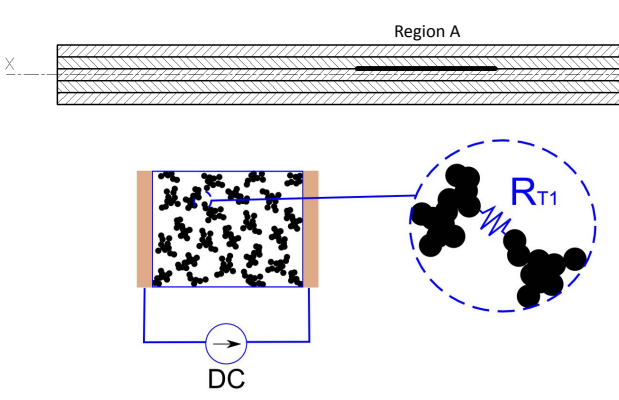

(a)

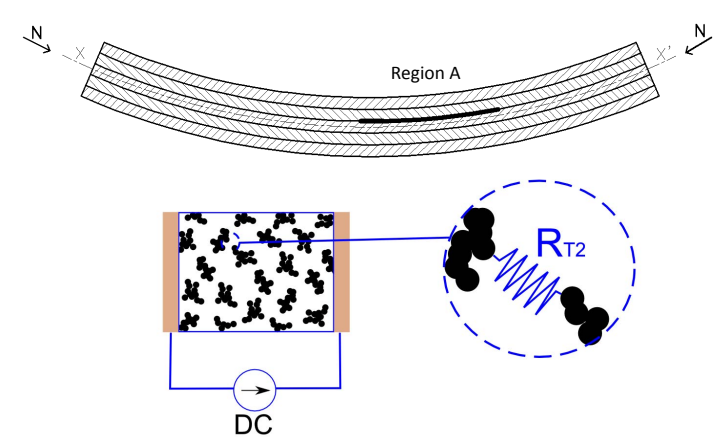

(b)

Figure 2. Tunneling effect is modelled by electrical resistance between CB conductive clusters. Under deformation the $\mathrm{CB}$ clusters increase the distance and hence the electrical resistance changes which alters the electrical paths. (a) Shows an initial configuration of CB clusters without any deformation and (b) presents changes on the CB clusters under a certain deformation.

The electrical conductivity can be explained and understood based on the percolation phenomenon [30]. A material can be conductive due to the formation of percolate electrical networks when it possesses isolated clusters of particles and the electric current is due to the tunnelling effect. The piezoresistivity is defined as the change of the electrical resistance caused by the change in the mechanical strain of the specimen. Then, the piezoresitivity can be measured by the change in the electrical resistance. The effect of the piezoresistivity has been studied under tensile strains [14], under compressive strains [12] and under vibratory excitation [8]. The piezoresistive properties in the nanoparticles can be attributed to two kinds of mechanisms which are able to introduce changes in the electrical resistance: 1) the loss of contact between the clusters of nanoparticles which can introduce considerable variation in the electrical conductivity and 2) the above mentioned tunneling effect which affects in the tunneling resistance due to the changes in the distances between the conductive particle clusters (see Figure 2). These two mechanisms affect the electrical conductivity and hence it alters the 
resistance which is measured in order to estimate the dynamic response.

Then, let consider a constant DC current through the material which follows an electrical path through the conductive particles along the specimen/beam. This electrical current provides a certain value of voltage which is proportional to the resistance defined by the electrical path chosen. Applying a mechanical load to the beam causes deformation and then because of the strain, the distances between the clusters of the conductive particles increase and therefore this introduces changes in the tunneling resistance between the clusters. The increment of this distance increases the tunneling resistance gradually. Therefore, the loss of contact or the changes of the tunneling resistance breaks the conductivity paths and forces the electrical current to choose an alternative path with smaller tunneling resistance. This phenomena occurs under small strains [31]. Additionally, it is important to mention that to obtain good piezoresistivity, it is better to include low fraction of conductive particles rather than high fraction as the probability to obtain tunneling effects is higher and hence more significant changes in the global resistance are measured [32].

Once a dynamic excitation is applied, it causes strain variations and the initial distribution of the nanoparticles along the specimen is altered. This alters the tunneling resistance and the electrical current path changes following the minimum resistance path. This behaviour provides variations in the global voltage measured between the both ends of the beam (see $\S 2$ ). The variations in the voltage are measured and recorded during the time of the dynamic excitation to obtain an estimate of the dynamic signal. In other words, the voltage measured through the conductivity of the CB-nanoparticles is used to estimate the dynamic signal. To verify this behaviour the following experiment is done. First, a constant DC is introduced through the beam and the voltage is then measured without any dynamic excitation. Secondly, the same beam is excited harmonically at $30 \mathrm{~Hz}$ by a shaker. For both cases the boundary conditions are kept constant by clamping one end of the beam in a cantilever configuration. More information about the test rig is given in $\S 5$. The two recorded signals are represented in the same Figure 3 and it can be observed that the $30 \mathrm{~Hz}$ harmonic due to the dynamic excitation can be clearly detected by the sharp peak at this frequency which does not appear for the case of no vibration.

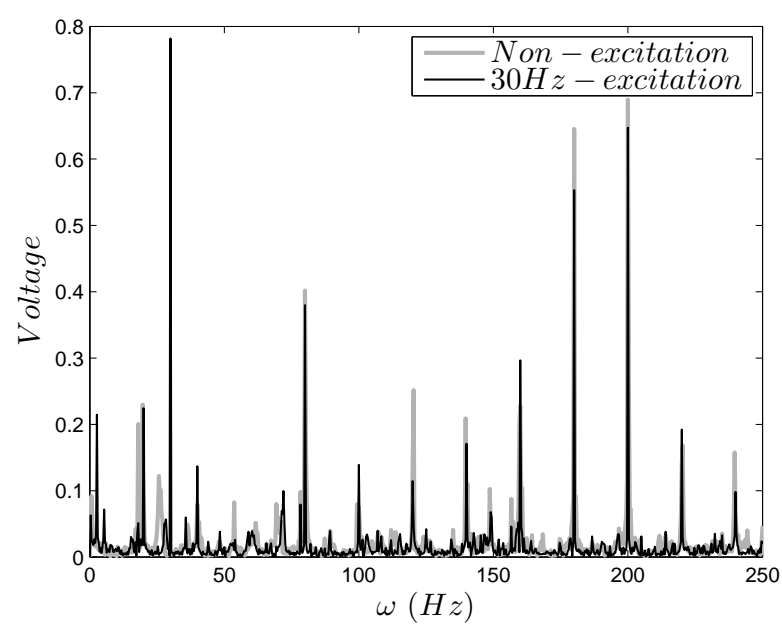

Figure 3. It can be observed the differences on the voltage measurements between a beam without any excitation (grey line) and the same beam harmonically at $30 \mathrm{~Hz}$ (black line).

As shown in Figure 3 the vibratory response can be measured through the voltage measurements of the CB filler. The voltage vibratory response characterises the health/damage state of the beam and any alteration due to damage or additional mass can be detected through 
it. Accordingly it can be concluded that the embedded nanoparticles provide self-sensing capabilities to the structure.

\section{Vibration-based damage assessment procedure}

This section presents the data driven vibration-based methodology for damage assessment which is applied on the voltage signals measured on the beams. The methodology is considered as a simple, nonparametric method for data compression and information extraction, which finds combinations of variables that describe major trends and oscillations in the voltage signals. The procedure is divided in four steps: data collection, creation of the reference state, feature extraction and damage assessment.

\subsection{Data collection}

The first step is to collect the data from the beams in consideration. The voltage signals were measured at each instant of time to obtain a signal for each realisation. Each signal is discretised into a vector with $\mathrm{N}$ data points equally spaced at $\Delta_{t}$ as shown in Equation 1

$$
\mathbf{x}_{m}=\left(x_{1, m}, x_{2, m}, \ldots, x_{N, i}\right)
$$

where $\mathrm{m}=1, \ldots, \mathrm{M}$ is the number of signal vector realisations. The signal vectors were normalised before applying any analysis to have zero mean and unity variance. The signal vectors for each m-realisation are arranged in columns into the matrix $\mathbf{X}$ with a dimension NxM.

$$
\mathbf{X}=\left(\mathbf{x}_{1}, \mathbf{x}_{2}, \ldots, \mathbf{x}_{M}\right)
$$

\subsection{Creation of the reference state}

The aim in this section is to create a reference state where the observation signal vectors can be compared. The reference state is based on the beam which will be considered as baseline which in this case is the healthy beam. The steps to create the reference state are: embedding, decomposition and reconstruction. These steps are explained as follows.

4.2.1. Embedding. This step creates an embedding matrix of the signal vectors. Dynamic systems cannot be fully unfolded in the two dimensional space of their measured signals because of their highly complex behaviour. By creating an embedding space, more dimensions are introduced and thus more features of the signal vector are uncovered. In this sense, each vector signal $\mathbf{x}_{m}$ is embedded into a matrix $\check{\mathbf{X}}_{m}$ by W-lagged copies of itself as shown in Equation 3 where $\mathrm{m}=1, \ldots, \mathrm{M}$ and $\mathrm{W}$ are the number of signal vector realisations and the sliding window size, respectively. The dimension of the matrix $\check{\mathbf{X}}_{m}$ is $\mathrm{N} \mathrm{x} \mathrm{W}$.

$$
\check{\mathbf{X}}_{m}=\left(\begin{array}{ccccc}
x_{1, m} & x_{2, m} & x_{3, m} & \cdots & x_{W, m} \\
x_{2, m} & x_{3, m} & x_{4, m} & \cdots & x_{(W+1), m} \\
x_{3, m} & x_{4, m} & x_{5, m} & \cdots & \vdots \\
x_{4, m} & x_{5, m} & \vdots & \cdots & \vdots \\
x_{5, m} & \vdots & \vdots & \cdots & x_{N, m} \\
\vdots & \vdots & x_{(N-1), m} & \cdots & 0 \\
\vdots & x_{(N-1), m} & x_{N, m} & \cdots & 0 \\
x_{(N-1), m} & x_{N, m} & 0 & \cdots & 0 \\
x_{N, m} & 0 & 0 & \cdots & 0
\end{array}\right)
$$


The embedding process defined in Equation 3 is applied on each signal vector realisation. Then, all matrices $\check{\mathbf{X}}_{m}$ are used to create the full embedding matrix $\check{\mathbf{X}}$. The dimension of the full embedding matrix $\check{\mathbf{X}}$ detailed in Equation 4 is $\mathrm{N} \times(\mathrm{MW})$. The number of M-signal vector realisations considered in the full embedding matrix $\check{\mathbf{X}}$ is normally selected so that $\mathrm{M} \leq \mathrm{W}$.

$$
\check{\mathbf{X}}=\left(\check{\mathbf{X}}_{1}, \check{\mathbf{X}}_{2}, \ldots, \check{\mathbf{X}}_{M}\right)
$$

4.2.2. Decomposition in Principal Components. This section explains the procedure to decompose the full embedding matrix $\check{\mathbf{X}} 4$ into a number of vector components based on of their variance content. First, the covariance matrix of $\check{\mathbf{X}}$ is calculated as detailed in Equation 5

$$
\mathbf{C}_{X}=\frac{\check{\mathbf{X}} \check{\mathbf{X}}^{t}}{N}
$$

The matrix $\mathbf{C}_{X}$ defines the covariance between the different signal vector realisations and has a dimension $(\mathrm{MW}) \mathrm{x}(\mathrm{MW})$. In the equation $5, \check{\mathbf{X}}$ is the full embedding matrix, $\check{\mathbf{X}}^{t}$ is the transpose matrix of $\check{\mathbf{X}}$ and $\mathrm{N}$ is the signal vector dimension. As the covariance matrix is calculated on the full embedding matrix, not only the auto-covariance of each signal vector realisation is considered but also the cross-covariance with respect to other signal vector realisations is also taken in to account.

The eigen-decomposition of $\mathbf{C}_{X}$ yields the eigenvalues $\boldsymbol{\lambda}_{k}$ and the eigenvectors $\boldsymbol{\rho}_{k}$ obtained by solving the following expression where the index $k$ represents each eigenvector and eigenvalue.

$$
\mathrm{C}_{X} \rho_{k}=\lambda_{k} \rho_{k}
$$

The eigenvalues $\boldsymbol{\lambda}_{k}$ are stored in the diagonal matrix $\boldsymbol{\Lambda}_{k}$ in decreasing order and the eigenvectors $\boldsymbol{\rho}_{k}$ are stored in columns into the matrix $\mathbf{E}_{X}$ in the same order than their corresponding eigenvalues. Each eigenvalue defines the partial variance in the direction of its corresponding eigenvector, therefore the sum of all eigenvalues gives the total variance of $\mathbf{X}$.

$$
\mathbf{E}_{X}^{t} \mathbf{C}_{X} \mathbf{E}_{X}=\Lambda_{X}
$$

The matrix $\mathbf{E}_{X}$ contains all eigenvectors $\mathbf{E}^{k}$ with dimension $\left\{\mathbf{E}^{k}: 1<k \leq M W\right\}$. Each eigenvector $\mathbf{E}^{k}$ is composed by $\mathrm{M}$ consecutive segments with a longitude $\mathrm{W}$. Therefore each element of an eigenvector is denoted as $E_{m, w}^{k}$. The Principal Component (PC) $\mathbf{A}_{k}$ associated to each $\mathbf{E}^{k}$, is calculated by projecting the $\check{\mathbf{X}}$ onto the $\mathbf{E}_{X}$ as shown in Equation 8 .

$$
A_{n}^{k}=\sum_{w=1}^{W} \sum_{m=1}^{M} X_{m, n+w} E_{m, w}^{k}
$$

Each element of the PC $A_{n}^{k}$ is a linear combination of the W-values of each M-segment weighted by their corresponding $\mathbf{E}^{k}$. Therefore, each PC contains characteristics from all the M signal vector realisations.

4.2.3. Reconstruction of the reference state. This section explains how to obtain the Reconstructed Components (RCs) which are linear combinations of the RCs and the eigenvectors. The RCs are calculated by convolving the PCs with the associated $\mathbf{E}^{k}$, thus the $k^{\text {th }} \mathrm{RC}$ at $n$-value for each $m$-realisation is given by the Equation 9 .

$$
R_{m, n}^{k}=\frac{1}{W_{n}} \sum_{w=1}^{W} A_{n-w}^{k} E_{m, w}^{k}
$$


Each $R_{m, n}^{k}$ value is normalised by the normalization factor $W_{n}$ which is described by the Equation 10.

$$
W_{n}= \begin{cases}n & 1 \leq n \leq W-1 \\ W & W \leq n \leq N\end{cases}
$$

The RCs are then arranged as columns into the matrix $\mathbf{R}$ with a dimension $\mathrm{N} \times \mathrm{MW}$. The matrix $\mathbf{R}$ includes all the RCs for the all the M-signal vectors and are distributed in sub-matrices as shown in Equation 11.

$$
\mathbf{R}=\left(\mathbf{R}^{1}, \mathbf{R}^{2}, \ldots, \mathbf{R}^{M}\right)
$$

Each of M-signal vector realisation is decomposed into W-reconstructed components arranged into the matrix $\mathbf{R}^{m}$ with a dimension $\mathrm{N} \mathrm{x} \mathrm{W}$ as shown in Equation 12.

$$
\mathbf{R}^{m}=\left(\begin{array}{ccc}
R_{1,1}^{m} & \cdots & R_{1, W}^{m} \\
\vdots & \ddots & \vdots \\
R_{N, 1}^{m} & \cdots & R_{N, W}^{m}
\end{array}\right)
$$

Then, $\mathbf{R}^{m}$ can be used as the reference state based on the healthy beam where the observation signal vectors are compared.

\subsection{Feature extraction}

In this section the procedure to obtain the feature vectors (FVs) is explained. A FV is obtained by multiplying an observation signal vector $\mathbf{x}$ with each of $\mathrm{W}$-columns of $\mathbf{R}^{m}$ as shown is Equation 13.

$$
T_{j}=\sum_{n=1}^{N} x_{n} R_{n, j}^{m}
$$

where $\mathbf{j}=1, . . \mathrm{W}$. Each $T_{j}$ value represents the inner product between an observation signal vector and each reconstructed component of $\mathbf{R}^{m}$. All $T_{j}$ are arranged into a vector $\mathbf{T}$ with dimension $\mathrm{W}$. The feature vector $\mathbf{T}$ characterises the observation signal vector onto a feature space with a dimension $\mathrm{W}$.

Therefore, when two feature vectors are compared onto the feature space, it is expected that if the two FVs are similar, the distance between them will decrease, however if they are different, the distance between them will increase.

\subsection{Damage assessment}

This section presents how damage is evaluated from the feature vectors defined in the section 4.3. First, a baseline feature space is created by a certain number of feature vectors $\mathbf{T}^{s}=$ $\left(T_{1, s}, T_{2, s}, \ldots, T_{p, s}\right)$ defined by the signal vectors from the healthy beam as shown in Equation 14.

$$
\mathbf{T}_{B}=\left(\begin{array}{cccc}
T_{1,1} & T_{2,1} & \cdots & T_{p, 1} \\
T_{1,2} & T_{2,2} & \cdots & T_{p, 2} \\
\vdots & \vdots & \cdots & \vdots \\
T_{1, s} & T_{2, s} & \cdots & T_{p, s}
\end{array}\right)
$$


In the above Equation $14, \mathbf{T}_{B}$ is the baseline $\mathrm{FV}$ matrix with a dimension $p \times \mathrm{x} s$ where $p$ is the dimension selected from the $\mathrm{FV}\{p \leq W\}$ and $s$ is the number of signal vectors utilised to define the baseline space. Once the baseline space is defined an observation FV is then compared with the baseline matrix $\mathbf{T}_{B}$. The observation FV must have the same dimension $p$ as the baseline matrix and is defined by $\mathbf{T}^{i}=\left(T_{1, i}, T_{2, i}, \ldots, T_{p, i}\right)$ where $i$ is the number of observation FVs.

The next step is to measure the similarity of an observation feature vector $\mathbf{T}^{i}$ to the set of baseline feature vectors $\mathbf{T}_{B}$. To demonstrate this, an outlier analysis using the Mahalanobis distance is carried out on the observation FVs. Outlier analysis calculates a measure of how similar or dissimilar an observation FV is to the baseline set. The measured distance is calculated as shown in Equation 15.

$$
D_{i}=\sqrt{\left(\mathbf{T}^{i}-\boldsymbol{\mu}_{B}\right)^{t} \boldsymbol{\Sigma}^{-1}\left(\mathbf{T}^{i}-\boldsymbol{\mu}_{B}\right)}
$$

In the above Equation 15, $\mathbf{T}^{i}$ is the observation $\mathrm{FV}, \boldsymbol{\mu}_{B}$ is the mean row of the baseline feature matrix $\mathbf{T}_{B}$ and $\boldsymbol{\Sigma}$ is the corresponding covariance matrix. In order to label an observation as an outlier or inlier there is a need to set a threshold value against a new distance can be evaluated. The threshold is calculated based on the distances measured for the baseline feature vectors $\mathbf{T}^{s}$ to the baseline matrix $\mathbf{T}_{B}$ as described in Equation 16.

$$
\vartheta=\mu_{D s}+\alpha \sigma_{D s}
$$

where $\mu_{D s}$ and $\sigma_{D s}$ are the mean and standard deviation of the Mahalanobis distances of $\mathbf{T}^{s}$ to $\mathbf{T}_{B}$ respectively and $\alpha$ is a constant to be determined. Therefore, the classification of a new $\mathrm{FV}$ is based on the comparison of $D_{i}$ to threshold described in Equation 16.

Two hypotheses are defined for FV classification as shown in Equation 17. H1 when the $D_{i}$ is equal or less than the threshold and $\mathrm{H} 2$ when the $D_{i}$ is greater than the threshold. In case of $\mathrm{H} 1$ the $\mathrm{FV} \mathbf{T}^{i}$ is assigned to the baseline category (inlier) while in case of $\mathrm{H} 2$ the $\mathrm{FV} \mathbf{T}^{i}$ is assigned as a non-baseline category (outlier).

$$
\begin{aligned}
& \text { H1 : } D_{i} \leq \vartheta \\
& \text { H2: } D_{i}>\vartheta
\end{aligned}
$$

Then, as the baseline is based on the healthy beam, any $D_{i}$ greater than the threshold is considered as an anomaly of the baseline and hence as a damaged beam.

\section{Experimental verification}

The following experiment was designed in order to verify the self-assessment properties of the considered nano-enriched laminates. The beam manufactured for this experiment, which is explained in $\S 2$, is subjected to simple harmonic vibration and to constant direct electric current. The voltage was measured at both ends of the beam as shown in Figure 4. The measured voltage signals were used as inputs to the damage assessment procedure explained in $\S 4.1$. Firstly for the study and comparison of the effect of the damage in the reference state and secondly for the damage assessment stage detailed in $\S 4.4$. The results are presented and discussed in $\S 6$.

\subsection{Experiment test rig}

The experiment rig to measure the voltage dynamic signals of the nano-enriched beam is shown in Figure 4. A constant electrical Direct Current was generated by Keithley 2140 Source-Meter and the voltage was measured with National Instruments 90253-Channel, $200 \mathrm{mV}$ to $10 \mathrm{~V}$, 
16-Bit Analog Input Module data acquisition (DAQ) card. Because of the high resistivity of the beam, a voltage buffer was constructed from OP-AMP TL07 to control the current leakage and hence to protect the overload of the DAQ. The vibration excitation was performed by a shaker and controlled by Laser vibrometer Polytec OFV-5000 and PCB accelerometer in order to apply exactly the same vibration excitation for all the experiments. The manufactured beam detailed in $\S 2$ was clamped in one end with a free span of $100 \mathrm{~mm}$. The clamp-support was perfectly connected to the excitation base of the shaker. This structure configuration guarantees the transmission of the vibration excitation to the composite laminated beam. Two electrodes were placed at both ends of the beam to guarantee a constant current along the entire beam and to allow voltage measurements through the material. Electrodes were prepared by first sanding the material in both edges. Secondly, a high conductive silver epoxy was applied on the surface and copper tape on top of the silver paint to design a permanent electrode as shown in Figure 5. Electrodes were manufactured carefully to reduce the additional effects on the measurements.

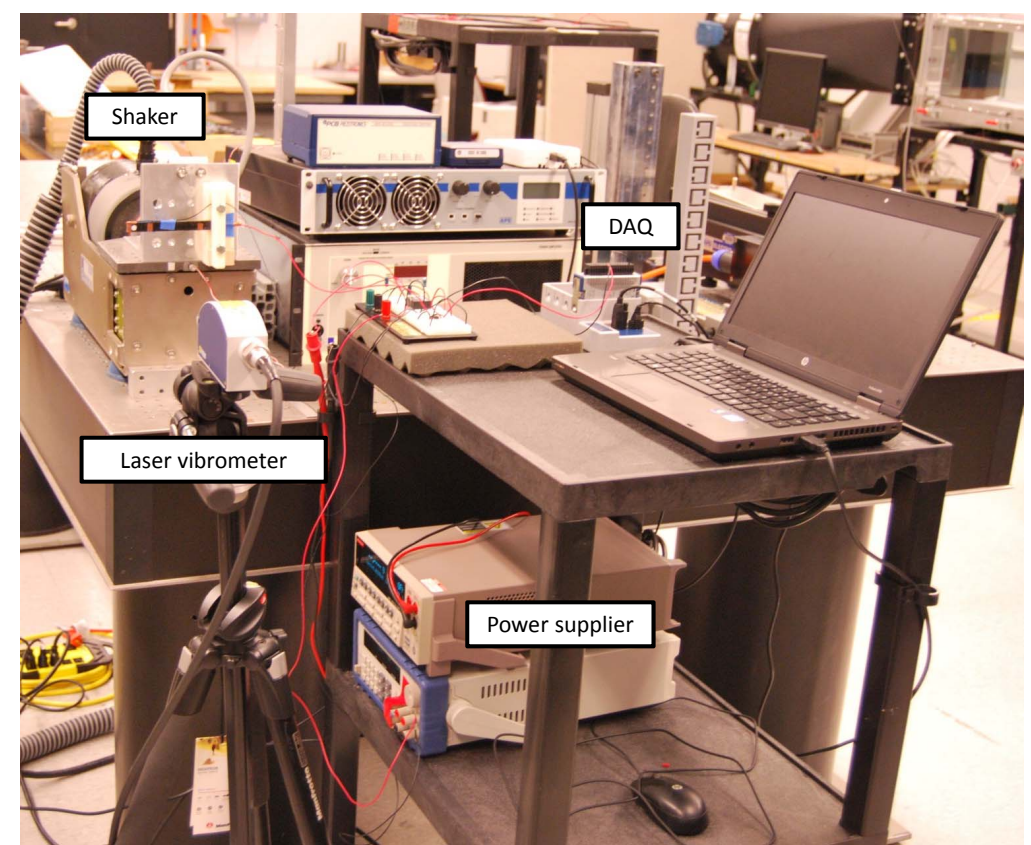

(a)

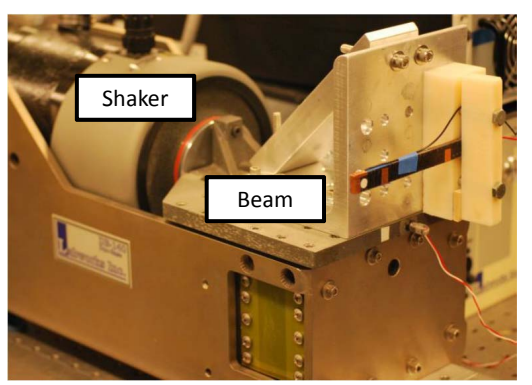

(b)

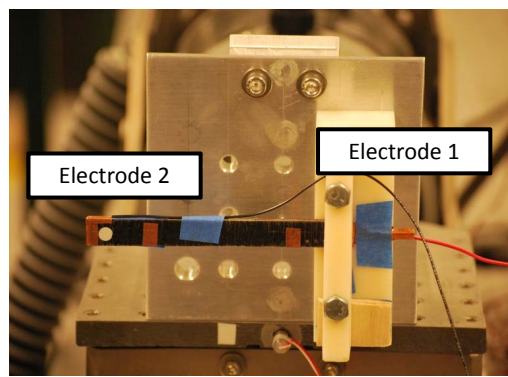

(c)

Figure 4. Test Rig. a) General picture of the test rig, b) how the beam is clamped on the shaker and c) the location of the two electrodes on the beam.

\subsection{Experiment procedure}

A constant DC current, lower than the limit imposed by the DAQ card, was applied on the beam (see Figure 5). Once a constant voltage can be measured through the beam, a simple harmonic excitation at $30 \mathrm{~Hz}$ by the shaker was applied. Vibration signals were estimated by measuring the voltage through the two electrodes. The amplitude/acceleration of the source excitation was controlled and configured to be constant for all the measurements in order to provide the same vibration excitation for each beam scenario (healthy and damage). Damage was introduced in two different manners: 1) Damage introduced by adding an additional mass on the tip of the beam and 2) Damage introduced by drilling a hole in the tip of the beam. For each damage configuration two damage scenarios were introduced 1) by adding two different masses ( $5 \%$ and $10 \%$ of the total mass of the beam respectively) and 2) by introducing two hole diameters (2 
$\mathrm{mm}$ and $4 \mathrm{~mm}$ respectively). Damages are introduced in the locations shown on Figure 6 . The damages were introduced without removing the beam from the test rig and hence the boundary conditions remained constant during the whole experiment. The idea is to avoid any influence of the support changes. For each beam scenario, 20 measurements were taken for $4 \mathrm{~s}$ at $500 \mathrm{~Hz}$. A $60 \mathrm{~Hz}$ bandstop filter was applied on the recorded signals to remove the presence of the Hum frequency. All the experiments were performed at room temperature.

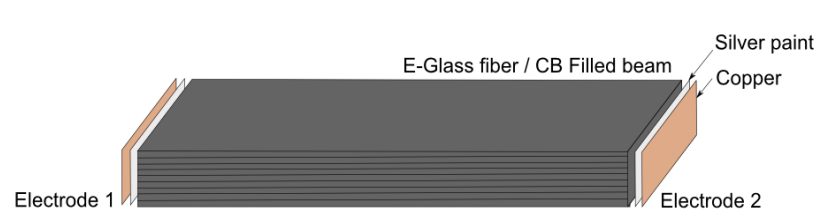

(a)

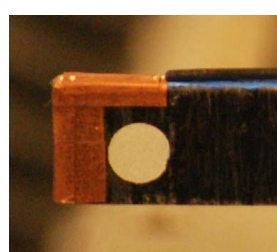

(b)

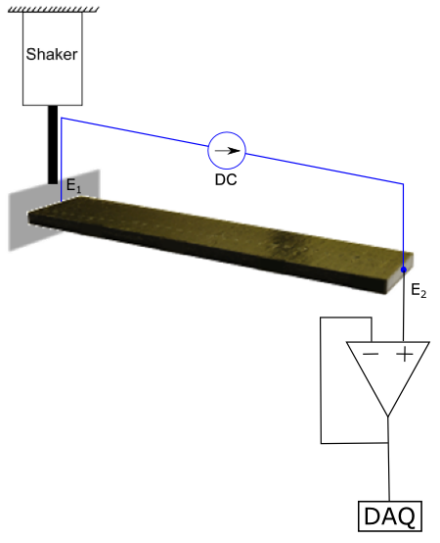

(c)

Figure 5. Electrode and measurement system. a) Silver epoxy material and copper tape were used to build the electrodes, b) final picture of an electrode and c) schematic picture of the measurements ring.

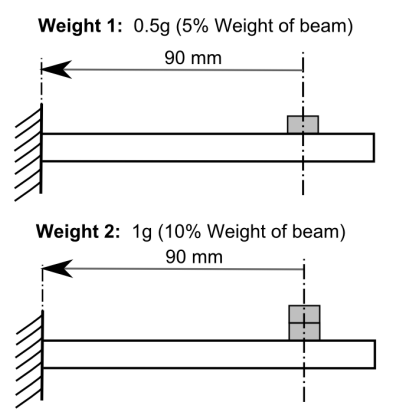

(a)

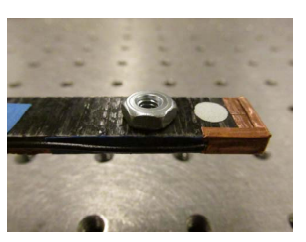

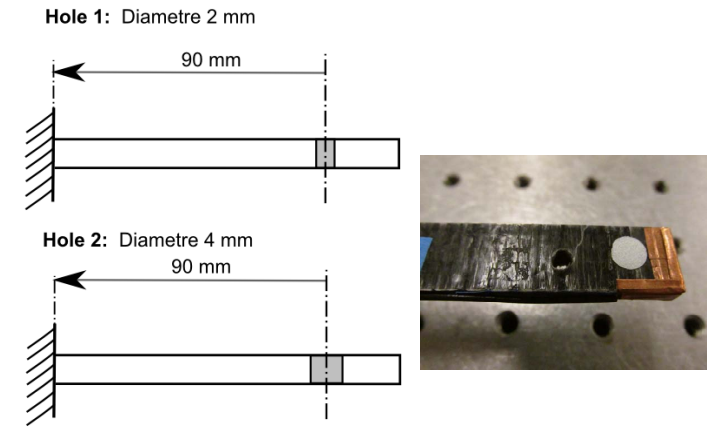

(b)

Figure 6. Description of the different damage introduced in the beam. a) Damage introduced by adding an additional mass and b) damage introduced by drilling a hole in the tip of the beam.

\section{Results and Discussions}

In this section the results are presented and discussed. First, the estimated vibratory responses from all beam categories described in Figure 6 are processed separately to obtain a reference state for each beam category as describes in $\S 4.2$. The reference state of each beam scenario is compared to see the effect of damage in the voltages signals. Secondly, the entire vibration-based damage assessment procedure detailed in $\S 4$ is applied on the estimated vibratory signals of the healthy beam to create a reference state. Then, the observation signals are compared to the baseline space for damage assessment as described in $\S 4.4$. 


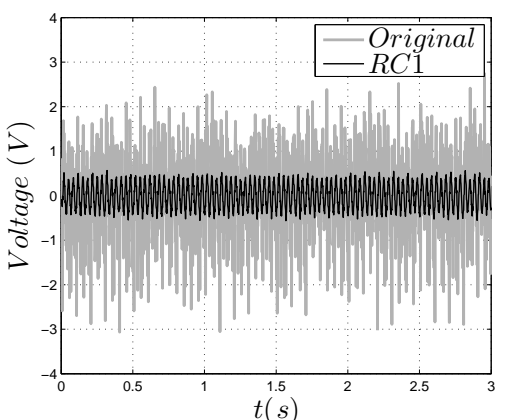

(a)

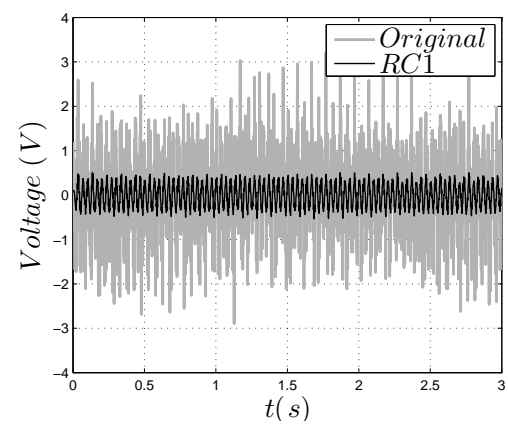

(b)

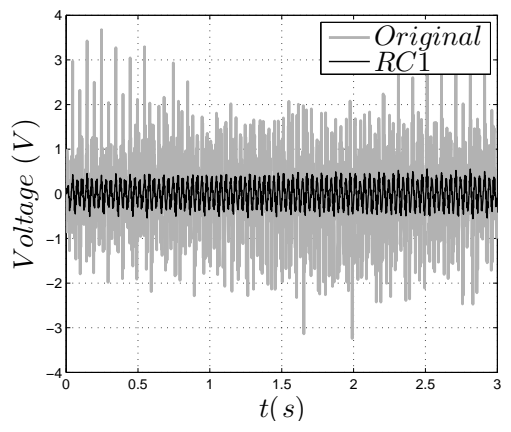

(c)

Figure 7. Comparison between the original raw voltage signal and the reconstructed signal by one $\mathrm{RC}$ from the beams with damage introduced by adding a mass. a) For healthy case (no mass added), b) for $0.5 \mathrm{~g}$ mass added ( $5 \%$ of the total mass) and c) for $1 \mathrm{~g}$ mass added ( $10 \%$ of the total mass).

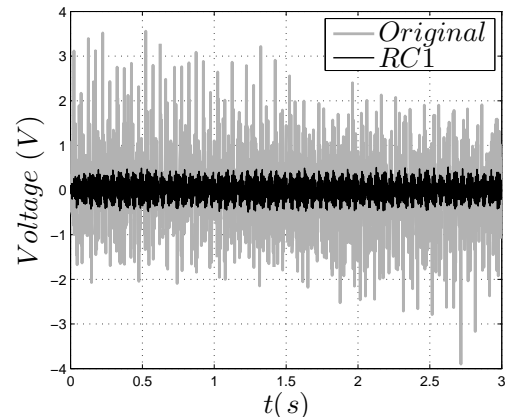

(b)

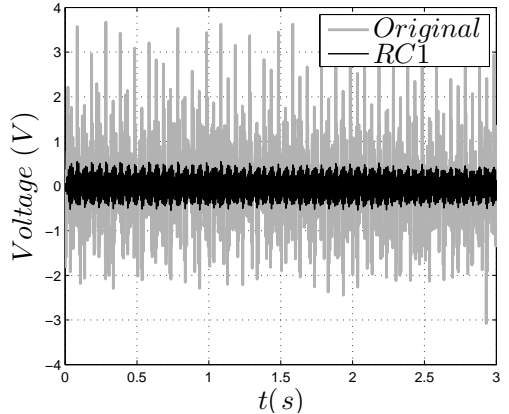

(c)

Figure 8. Comparison between the original raw dynamical signal and the reconstructed signal by one RC from the beams with damage introduced by drilling a hole. a) For healthy case (no hole drilled), b) for a $2 \mathrm{~mm}$ hole drilled and c) for a $4 \mathrm{~mm}$ hole drilled. 
The frequency spectrum of the beam with added mass and the one with hole drilled are compared in Figure 9. It can be observed that for the case of the beam damaged by a hole, higher peaks appear at higher frequencies as compared to the peaks of the beam with mass added. However, in both cases the $30 \mathrm{~Hz}$ frequency is present in the reconstructed signals as shown in Figures 9(a) and 9(b). The peaks at higher frequencies between 180 and $200 \mathrm{~Hz}$ are due to the electrical conductivity and peaks appear because of the harmonics of the hum frequency. This is very well depicted in the frequency spectrum of signals from the beam with a hole drilled (see Figure 9(b)) because in this case not only the beam is damaged but also the CBnanocomposites embedded through the laminates. The hole reduces the electrical conductivity paths and as a result the correspondence/relation between the dynamic and the voltage signals is reduced. This finding is also coherent with the results obtained in [9] where a hole-drilled in a CB-nanoparticle enriched laminated was detected by changes in the voltage measured in the edge electrodes within the specimen domain.

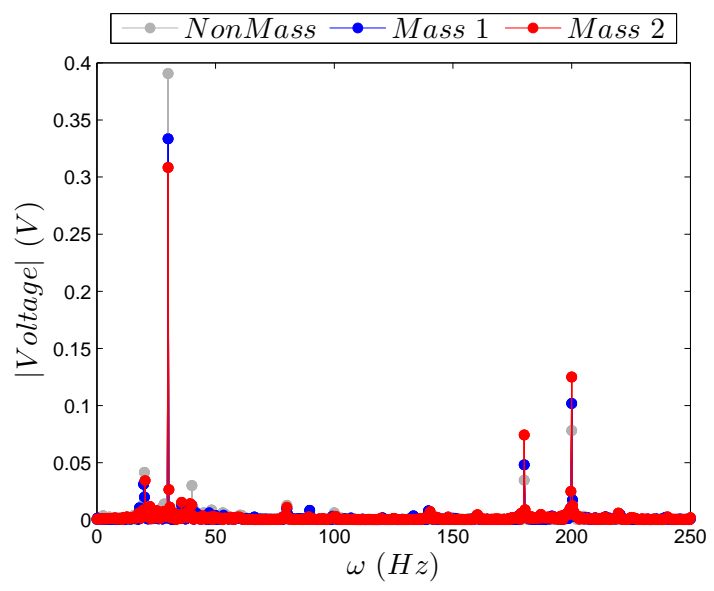

(a)

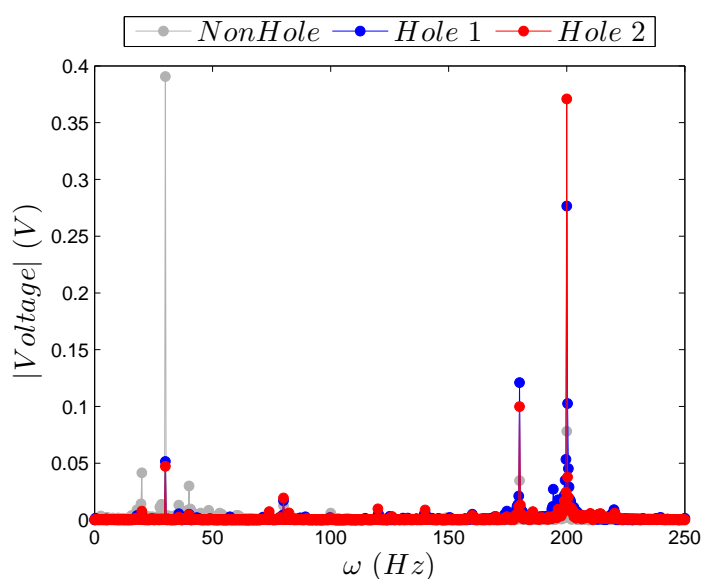

(b)

Figure 9. Comparison of the frequency spectrum of the first reconstructed component for a) the case of the beam with damage introduced by adding a mass and b) the case of the beam with damage introduced by drilling a hole.

Figures 10(a) and 10(b) present the phase maps which can be estimated by plotting the first two RCs from the voltage signal decomposition [35]. Each plot contains three signals: one from the healthy beam and others two corresponding to the different damage scenarios. Time series are presented in the 2-dimensional space reconstructed from the first two reconstructed components, RC1 and RC2. Figure 10 shows that the areas covered by the first two RCs for the different damage scenarios are well distinguishable. The one for the healthy voltage signal is shifted with regards to the damaged ones and the two damaged signals are also shifted with respect to each other. As it is shown in [8], changes in amplitude were found in the vibration response obtained by carbon nanotube strain sensor when the responses were measured in cantilever beam with and without a crack induced. This is also coherent with the results, where the voltage signal measurements can provide indication for the presence of damage and they can be used to distinguish between the healthy and the two damage scenarios. Changes in the areas corresponding to the beam with a hole are less observable as compared to the changes for the added mass. This can be explained again by the damaged conductivity paths which affect the relation between the dynamic and the voltage signal (see Figure 10(b)). 


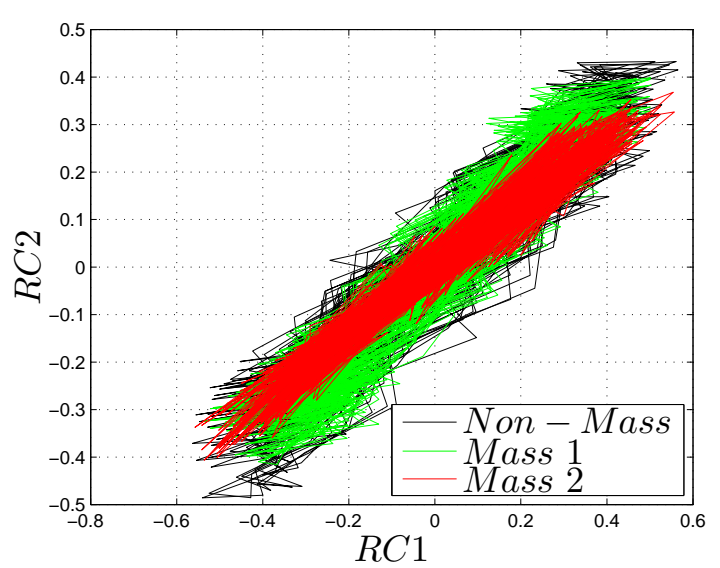

(a)

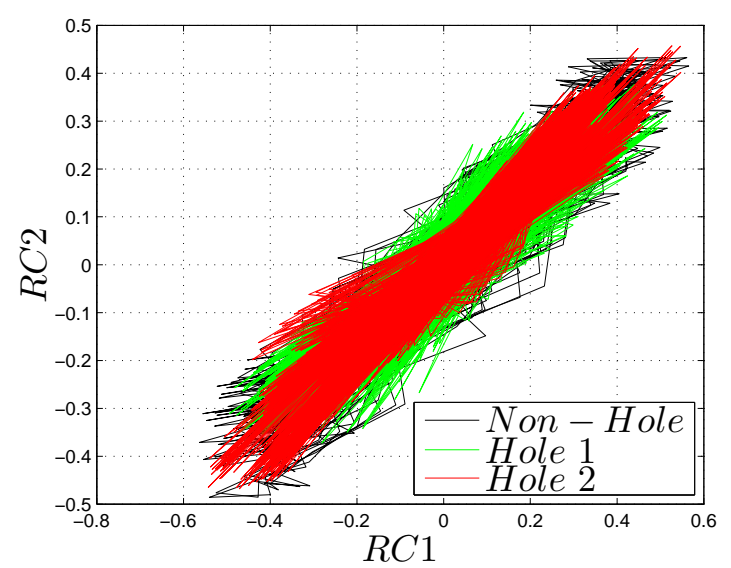

(b)

Figure 10. Comparison of the phase portraits of the time series using RC1 and RC2 for the case of a) the beam with damage introduced by adding a mass and b) the beam with damage introduced by drilling a hole.

\subsection{Damage assessment on the composite laminated beams}

As presented in $\S 6.1$, it is demonstrated that the signals obtained from the voltage measurements between the two electrodes on the beam can provide indication for the presence of damage and hence be used for damage assessment. Therefore, the voltage signals are processed by the entire methodology as described in $\S 4$. The same procedure has been applied for the case of the beam with mass added and the beam with a hole drilled. The sliding window size was considered at $\mathrm{W}=10$ and the number of vector signal realisations to build the reference state was $\mathrm{M}=5$ from the healthy beam.

For visualisation purposes, it can be observed that the projection of the observation FVs onto a two dimensional feature space generates different clusters based on the similarity between the observation FVs. The FVs obtained from the same category reduce their distances between themselves when are projected onto the feature space while they increase their distances to FVs from other categories. These clusters can be observed in Figures 11(a) and 12(a) for the beam with damage introduced by adding a mass on the tip and for the beam with a damage introduced by drilling a hole in the tip respectively. Then, a baseline space is built based on the FVs of the healthy beam (without any mass or hole introduced) where each observation FV can be compared. The distance of each observation $\mathrm{FV}$ to the baseline is measured as shown in Equation 15. As explained in 4.4, a threshold is calculated based on the distances of FVs utilised to build the baseline respect to the baseline itself. Therefore, the distances from new observation FVs are compared to this threshold for damage assessment. A distance point below the threshold is considered as a FV obtained from the healthy beam while a distance over the threshold is considered as a FV obtained from a damage beam. Figures 11(b) and 12(b) show clearly that the FVs obtained from damaged beams increase their distances with respect to the baseline threshold and hence the points are placed over the threshold dashed line. On the other hand, FVs from healthy beam observations have distances that are smaller than the threshold and hence they are considered as observations from the healthy beam. It is also important to mention, that the severity of the damage cannot be monitored because the distances does not increase by increasing the severity of the damage. This behaviour occurs in the both damages scenarios having the same trend for the beam with mass added and for the beam with a hole drilled. However damage is successfully detected for the both damage scenarios because all the distances measured from observation FVs of damaged beams are greater than the threshold. 
Then, it is clear that damage is successfully detected for both cases but the damage index used cannot be used to infer severity from the measured features.

As mentioned above in $\S 6.1$ the sensitivity for damage detection in the case of the beam with a hole drilled is smaller than for the case of mass added. It is then observed that the distances are smaller in comparison with the case of mass added. However, for both damage sizes the detection is successfully achieved.

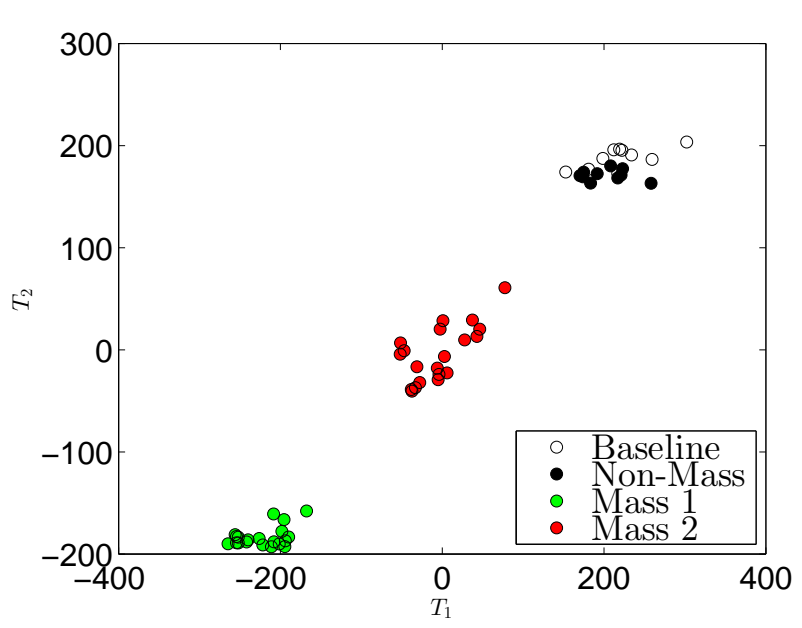

(a)

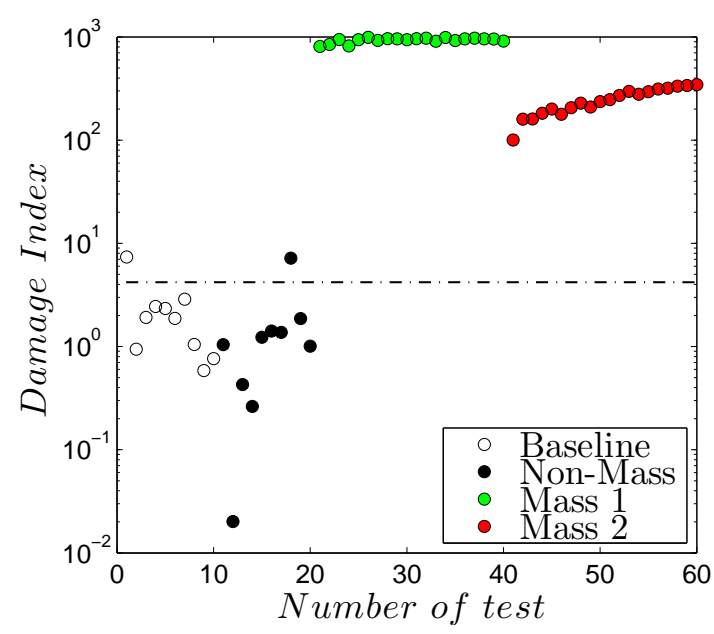

(b)

Figure 11. For the case of beam with damage introduced by adding a mass a) clusters obtained in a 2-dimensional feature space and b) Mahalanobis distances of the different damages scenarios to the healthy scenario.

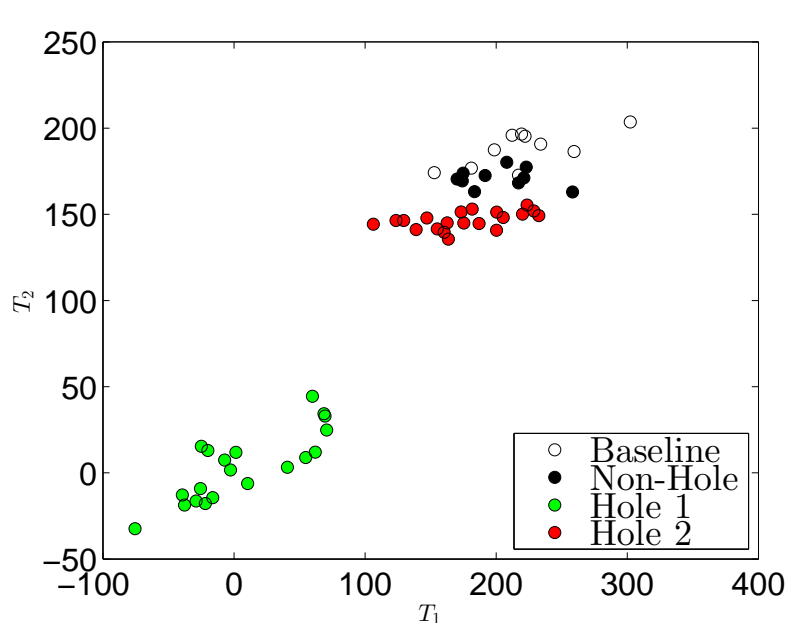

(a)

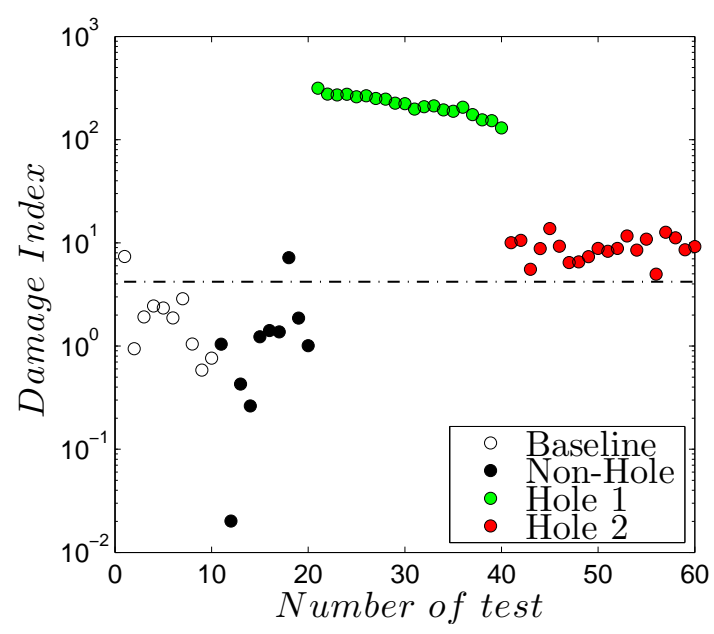

(b)

Figure 12. For the case of beam with damage introduced by drilling a hole a) clusters obtained in a 2-dimensional feature space and b) Mahalanobis distances of the the different damages scenarios to the healthy scenario. 


\section{Conclusions}

This study explores the self-sensing capabilities of a beam manufactured with CB nano-particles embedded between the glass/fibre laminates. The voltage is measured through the beam due to the electrical conductivity capabilities of the CB nano-particles. The dynamic strain introduced in the beam changes the CB nano-particles clusters and hence changes in the electrical resistance are introduced. Voltage signals are measured to construct estimated vibratory responses.

A data driven methodology is utilised for features extraction by the decomposition into a number of reconstructed components of the voltage signals measured of the healthy beam. The new reconstructed signal vectors are used to create a reference state where new observation vectors are compared and eventually compared for the purposes of self-damage assessment. This analysis has been performed for two different beam categories. Firstly, the damage has been introduced by adding one and two masses (5\% and $10 \%$ of the total mass of the beam) and secondly by drilling two different hole sizes ( $2 \mathrm{~mm}$ and $4 \mathrm{~mm}$ respectively) near the tip of the beam. The damage detection is successfully achieved for both cases. The main conclusions of this study are highlighted as follow:

- Vibratory signals can be estimated by measuring changes in voltage due to the electromechanical properties of the CB nano-particles embedded in a glass/fibre laminated beam.

- The voltage signal measurements can provide indication for the presence of damage and they can be used to distinguish between the two damage scenarios.

- The voltage signal responses were processed by a vibration-based structural health monitoring methodology which is able to extract features qualifying the state/health of the beam, which eventually results in the self-damage assessment.

Based on the results, it is demonstrated that conductive composite laminates have substantial potential for self-sensing capabilities. The results open a new path of investigation towards vibration self-sensing structural health monitoring with nanocomposites inclusions. Further work must be done in the configuration and distribution of the nanocomposites for reducing the high resistivity of the material, as well as the development of novel methodologies to obtain the most from the measurements.

\section{Acknowledgments}

The collaboration support provided by The Royal Society of Edinburgh through John Moyes Lessells Travel Scholarship, by The Institution of Mechanical Engineers through the Flatman grant and by The Institution of Engineering and Technology Travel Award is gratefully acknowledge by the authors. The authors also wish to acknowledge the help of Dr Tyler Tallman for providing the composite laminate beams used for the experiments. The authors also acknowledges the useful discussions and the support during the experiments provided by Dr. Jared Hobeck. This investigation would not be have been possible without the continuous and invaluable support of the Department of Aerospace Engineering at The University of Michigan and the Mechanical and Aerospace Engineering Department at The University of Strathclyde.

\section{References}

[1] Thostenson E T and Chou TW, 2006. Carbon nanotube networks: sensing of distributed strain and damage for life prediction and self healing. Advanced Materials. 18(21):2837-2837

[2] Ubertini F, Laflamme S, Ceylan H, Materazzi An L, Cerni G, Saleem H, D'Alessandro A and Corradini A, 2014. Novel nanocomposite technologies for dynamic monitoring of structures: a comparison between cement-based embeddable and soft elastomeric surface sensors. Smart Materials and Structures. 23(4):045023

[3] Brook I, Mechrez G, Suckeveriene RY, Tchoudakov R, Lupo S and Narkis M, 2014. The structure and electromechanical properties of novel hybrid CNT/PANI nanocomposites. Polymer Composites. 35(4):788-794 
[4] Dharap P, Li Z, Nagarajaiah S and Barrera EV, 2004. Nanotube film based on single-wall carbon nanotubes for strain sensing. Nanotechnology. 15(3):379

[5] Todoroki A, Yoshida J, 2004. Electrical resistance change of unidirectional CFRP due to applied load. JSME International Journal Series A. 47(3):357-364

[6] Saghafi H, Zucchelli A, Palazzetti R and Minak G, 2014. The effect of interleaved composite nanofibrous mats on delamination behavior of polymeric composite materials. Composite Structures. 109:41-47

[7] Abot JL, Song Y, Vatsavaya MS, Medikonda S, Kier Z, Jayasinghe C, Rooy N, Shanov VN and Schulz MJ, 2010. Delamination detection with carbon nanotube thread in self-sensing composite materials. Composites Science and Technology. 70(7):1113-1119

[8] Kang I, Schulz MJ, Kim JH, Shanov V and Shi D, 2006. A carbon nanotube strain sensor for structural health monitoring. Smart materials and structures. 15(3):737

[9] Tallman T N, Gungor S, Wang K W and Bakis C E, I, 2015. Damage detection via electrical impedance tomography in glass fiber/epoxy laminates with carbon black filler. Structural Health Monitoring. 14(1):100-109

[10] Hou T-C, Loh KJ, Lynch JP, 2007, Spatial conductivity mapping of carbon nanotube composite thin films by electrical impedance tomography for sensing applications. Nanotechnology. 18(31):315-501

[11] Loyola BR, Briggs TM, Arronche L, Loh KJ, La Saponara V, O'Bryan G and Skinner JL, 2013, Detection of spatially distributed damage in fiber-reinforced polymer composites. Structural Health Monitoring. 12(3):225-239

[12] Loh KJ, Lynch JP, Shim BS, Kotov NA, 2008, Tailoring piezoresistive sensitivity of multilayer carbon nanotube composite strain sensors. Journal of Intelligent Material Systems and Structures. 19(7):747-764

[13] Hu N, Karube Y, Yan C, Masuda Z, Fukunaga H, 2008. Tunneling effect in a polymer/carbon nanotube nanocomposite strain sensor. Acta Materialia. 56(13):2929-2936.

[14] Pham G T, Park Y-B, Liang Z, Zhang C and Wang B, 2008. Processing and modeling of conductive thermoplastic/carbon nanotube films for strain sensing. Composites Part B: Engineering. 39(1):209-216.

[15] Doebling S W, Farrar C R, Prime M B, Shevitz D W, 1996. Damage identification and health monitoring of structural and mechanical systems from changes in their vibration characteristics: a literature review. Los Alamos National Lab., NM (United States)

[16] Zou Y, Tong LPSG, Steven GP, 2000. Vibration-based model-dependent damage (delamination) identification and health monitoring for composite structuresa review. Journal of Sound and vibration 230(2):357-378

[17] Montalvao D, Maia NMM and Ribeiro AMR, 2006. A review of vibration-based structural health monitoring with special emphasis on composite materials. Shock and Vibration Digest 38(4):295:326

[18] Cawley $\mathrm{P}$ and Adams RD, 1979. The location of defects in structures from measurements of natural frequencies. The Journal of Strain Analysis for Engineering Design. 14(2):49-57.

[19] Pardoen GC, 1989. Effect of delamination on the natural frequencies of composite laminates. Journal of composite materials. 23(12):1200-1215.

[20] Trendafilova I, Palazzetti R and Zucchelli A, 2015. Damage assessment based on general signal correlation. Application for delamination diagnosis in composite structures. European Journal of Mechanics-A/Solids. 49():197-204.

[21] Marin L, Döhler M, Bernal D and Mevel L, 2014. Robust statistical damage localization with stochastic load vectors. Structural Control and Health Monitoring. 22(3):557-573.

[22] Sakaris CS, Sakellariou JS and Fassois SD, 2015, Vibration-based damage precise localization in threedimensional structures: Single versus multiple response measurements. Structural Health Monitoring. 14(3):300-314

[23] Garcia D and Trendafilova I, 2014, A multivariate data analysis approach towards vibration analysis and vibration-based damage assessment: Application for delamination detection in a composite beam. Journal of Sound and Vibration. 333(25):7036-7050

[24] Chao, S-H and Loh C-H, 2014, Application of singular spectrum analysis to structural monitoring and damage diagnosis of bridges. Structure and Infrastructure Engineering. 10(6):708-727

[25] Rubin, Z and Sunshine, SA and Heaney, MB and Bloom, I and Balberg, I, 1999. Critical behavior of the electrical transport properties in a tunneling-percolation system. Physical Review B. 59(19):12 196-12 199

[26] Gungor, S and Bakis, C E, 2014. Anisotropic networking of carbon black in glass/epoxy composites using electric field Journal of Composite Materials

[27] Li ZH, Zhang J and Chen SJ, 2008. Effects of carbon blacks with various structures on vulcanization and reinforcement of filled ethylene-propylene-diene rubber. Express Polymer Letters. 2(10):695-704.

[28] Oskouyi A B, Sundararaj U and Mertiny P 2014. Tunneling conductivity and piezoresistivity of composites containing randomly dispersed conductive nano-platelets. Materials. 7(4):2501-2501.

[29] Jing X, Zhao W and Lan L, 2000, The effect of particle size on electric conducting percolation threshold in polymer/conducting particle composites. Journal of materials science letters. 19(5):377-379 
[30] Kwon G, Heo Y, Shin K and Sung, B J, 2012, Electrical percolation networks of carbon nanotubes in a shear flow. Physical Review E. 85(1):011143

[31] Theodosiou TC and Saravanos DA, 2010. Numerical investigation of mechanisms affecting the piezoresistive properties of CNT-doped polymers using multi-scale models. Composites Science and Technology. 70(9):1312-1320.

[32] Hu N, Fukunaga H, Atobe S, Liu Y and Li J, 2011. Piezoresistive strain sensors made from carbon nanotubes based polymer nanocomposites. Sensors 11(11):10691-10723

[33] Garcia D, and Trendafilova I, 2014. Singular spectrum analysis for identifying structural nonlinearity using free-decay responses: Application detection and diagnosis in composite laminates. Proceedings of 26th International Conference on Noise and Vibration Engineering

[34] Loh K J, Kim J and Lynch J P, 2008. Self-sensing and power harvesting carbon nanotube composites based on piezoelectric polymers Proceedings of the 4 th International Conference on Bridge Maintenance, Safety and Management 3329-3336.

[35] Broomhead S and King G P, 1986. Extracting qualitative dynamics from experimental data. Physica D: Nonlinear Phenomena 20(2):217-236 